\title{
REVIEW
}

\section{Classification and grading of the non-invasive urothelial neoplasms: recent advances and controversies}

\section{R Montironi, A Lopez-Beltran, R Mazzucchelli, D G Bostwick}

J Clin Pathol 2003;56:91-95

The classification and grading of the non-invasive, intraepithelial neoplasms of the urothelium are based on the morphological pattern of growth - that is, papillary or flat (and endophytic) - and on their degree of architectural and cytological abnormalities. Recent advances in the morphological, molecular, and quantitative evaluation of these lesions have contributed to the refinement of the current classification and grading schemes. However, some controversies on the precise criteria and terminology, especially when the papillary lesions are concerned, are still present.

See end of article for authors' affiliations

Correspondence to: Professor R Montironi, Institute of Pathological Anatomy and

Histopathology, University of Ancona School of Medicine, Umberto $1^{\circ}$ Hospital, I-60020 Torrette, Ancona, Italy;

r.montironi@unian.it

Accepted for publication 30 September 2002
$\mathrm{E}$ arly clinical observations regarding the biology of the "at risk" field suggested that sites of urothelial preneoplastic changes could follow several distinct clinical courses ( fig 1). ${ }^{1}$ It is possible that areas of dysplasia remain simply dysplastic. Alternatively, the urinary epithelium can progress either to superficial bladder neoplasm, characterised by recurrence but rare life threatening progression, or along the path towards invasion, with its well recognised risk of mortality. Evidence in support of these disparate pathways comes from the low progression rate of most superficial bladder tumours, coupled with that fact that many invasive neoplasms present as such initially. ${ }^{2}$

From the morphological point of view, two basic pathways are identified on the basis of the pattern of growth of the intraepithelial lesions (papillary and flat), the behaviour of these lesions being related to the degree of architectural and cytological alteration of the urothelium. Several classification and grading schemes (including revisions and refinements) of urothelial noninvasive or intraepithelial lesions have been reported in the literature. ${ }^{2-7}$

\section{"Two basic pathways are identified on the basis of the pattern of growth of the intraepithelial lesions (papillary and flat), the behaviour of these lesions being related to the degree of architectural and cytological alteration of the urothelium"}

The aim of our review is to give an overview of recent advances and controversies in the classification and grading of the non-invasive, intraepithelial urothelial papillary and flat neoplasms

\section{INTRAEPITHELIAL PAPILLARY LESIONS WHO 1973 classification}

The classification of the World Health Organisation (WHO) published in 1973 (WHO 1973) includes urothelial papilloma and three grades of papillary carcinoma (grade 1, or G1; grade 2, or G2; and grade 3, or G3). ${ }^{3}$ Urothelial carcinoma grade correlates with the probability of recurrence, invasion, and metastasis, in addition to survival. ${ }^{7-11}$

Urothelial papilloma is defined as a small usually solitary papillary lesion, with a delicate fibrovascular core lined by cytologically and architecturally normal urothelium without mitotic figures (Appendix). ${ }^{37}$ Mild cytological atypia of the superficial cells does not exclude the diagnosis of papilloma, particularly when accompanied by an explanatory inflammatory infiltrate.

\section{"The morphological criteria useful for classification and grading have been continuously refined and updated"}

The grading of papillary urothelial carcinoma is based on the worst grade present. Gl carcinoma consists of a urothelium more than seven cell layers thick containing cells that display minimal to slight nuclear enlargement, normal or slightly distorted architecture, and rare or absent mitotic figures. In contrast, G2 carcinoma displays greater nuclear pleomorphism, coarsely clumped chromatin, and some disruption of the normal architecture. Malmstrom et al subdivided G2 into G2A and G2B in 1987. ${ }^{12}$ Morphologically, G1 and G2A are similar to urothelial flat dysplasia (see below). Grade 3 carcinoma displays the most extreme nuclear abnormalities, similar to those seen in carcinoma in situ (CIS). Cellular anaplasia, characteristic of grade 3 carcinoma, is defined as increased cellularity, nuclear crowding, disturbance of cellular polarity, absence of differentiation from the base to the mucosal surface, nuclear pleomorphism, irregularity in the size of the cells, variation in nuclear shape and chromatin pattern, increased number of mitotic figures, and the occasional presence of neoplastic giant cells. ${ }^{3}$

The morphological criteria useful for classification and grading have been continuously refined and updated. ${ }^{13}{ }^{14}$ Recent efforts to grade

Abbreviations: CIS, carcinoma in situ; G, grade; ISUP, International Society of Urological Pathology; $\mathrm{LOH}$, loss of heterozygosity; PUNLMP, papillary urothelial neoplasm of low malignant potential; WHO, World Health Organisation 


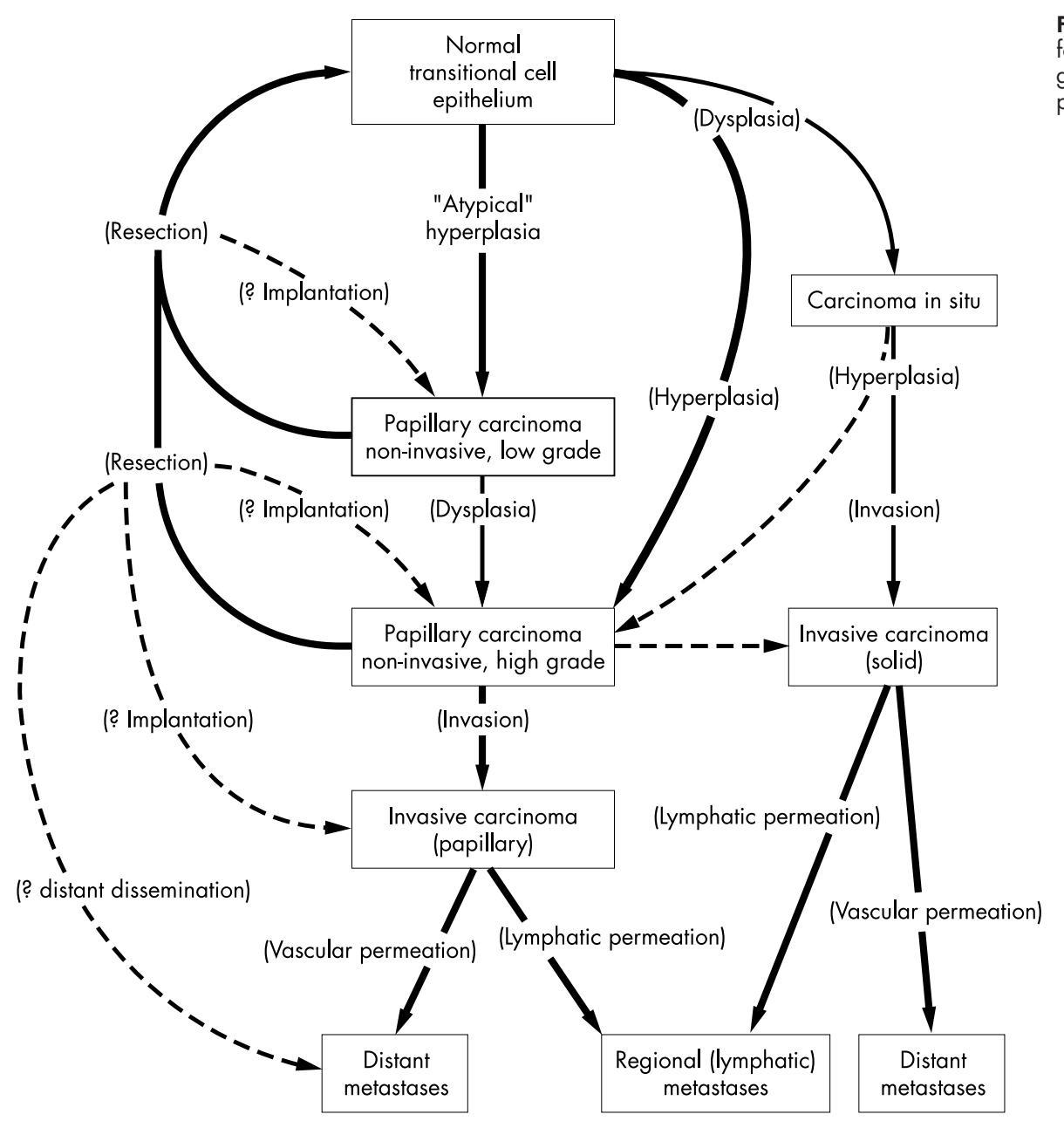

Figure 1 Morphological pathways for bladder tumour classification and grading. Reproduced, with permission, from Jones et al.'

urothelial carcinoma using image analysis based on nuclear morphometry, silver staining nucleolar organiser regions, diagnostic decision support systems (Bayesian belief networks), and other markers have also been successful, but are not used routinely. ${ }^{13}{ }^{16}$ This has led to a high degree of reproducibility and accuracy in the classification and grading of papillary neoplasms. ${ }^{13}$

\section{WHO/ISUP 1998 and WHO 1999 classifications}

The WHO/International Society of Urological Pathology (ISUP) consensus classification of 1998 (WHO/ISUP 1998) distinguishes between papilloma, papillary urothelial neoplasm of low malignant potential (PUNLMP), and low and high grade carcinoma." The term "papillary urothelial neoplasm of low malignant potential" was introduced to replace WHO 1973 Gl carcinoma in recognition of the low probability of recurrence or progression of this neoplasm, especially after complete removal, and the preference not to label these patients with the term "cancer". ${ }^{45}$ The WHO classification introduced in 1999 (WHO 1999) is almost identical to the WHO/ISUP classification, the difference being that the WHO 1999 scheme subdivides the low and high grade spectrum into three grades (grades I, II, and III). ${ }^{6}$

\section{WHO 1973 versus WHO/ISUP 1998 and WHO 1999: controversies}

The topic of the best contemporary classification of the papillary neoplasia was debated at the Ancona international consultation on the diagnosis of non invasive urothelial neoplasms (11-12 May 2001, Ancona, Italy). ${ }^{17}$ The discussion was basically around the WHO 1973 scheme versus the WHO/
ISUP 1998 and WHO 1999 classifications. In particular, the following problems were considered:

- Are detailed morphological criteria available for the WHO 1973 scheme and for the WHO/ISUP 1998 and WHO 1999 classifications?

- Reproducibility: is reproducibility better with the WHO/ ISUP 1998 and WHO 1999 classifications?

- Gl papillary carcinoma (1973 scheme): is this a misnomer for a lesion that does not have the morphological and clinical features of cancer? Should the term "papillary neoplasia with low malignancy potential" be used to avoid the term carcinoma?

- Translation from the WHO 1973 scheme to the WHO/ISUP 1998 and 1999 WHO classifications: is it feasible and easy?

- How much clinical and prognostic information is available in favour of the WHO 1973 scheme and how much in favour of the WHO/ISUP 1998 and WHO 1999 classifications?

A full consensus on which classification should be used by practising pathologists and followed by urologists and oncologists was not reached. Drs Bostwick and Mikuz ${ }^{3}$ represented the majority opinion-for example:

- The WHO 1973 classification for papillary urothelial neoplasms was still superior to all existing alternatives (including WHO/ISUP 1998 and WHO 1999), although some refinement of diagnostic criteria would be useful.

- Some pathologists may prefer to report additional, synonymous classifications in other schemes, but this is discouraged owing to variations and difficulties in translation. 


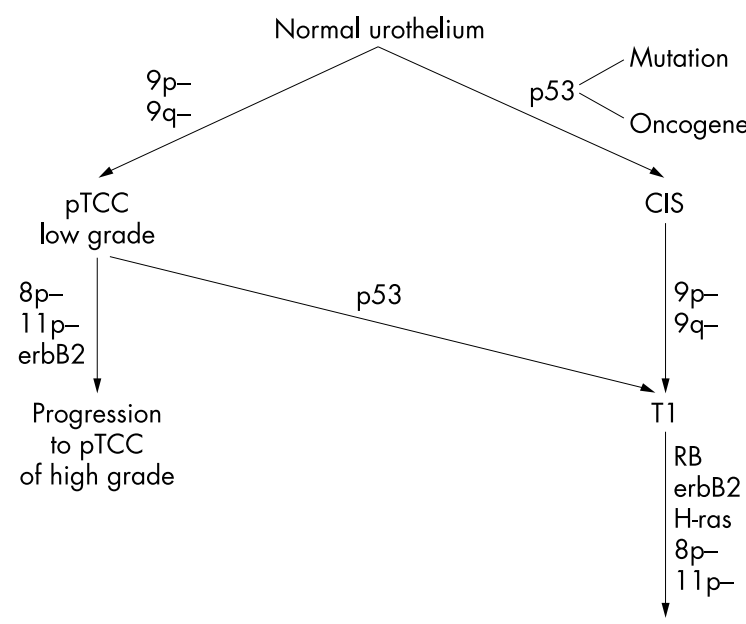

Progression

to T2-4 and metastases

Figure 2 Molecular and genetic based pathways for bladder tumour classification and grading. Reproduced, with permission, from Brandau et al. ${ }^{47}$

Drs C Busch and F Algaba advocated the contemporary role of the WHO/ISUP 1998 and WHO 1999 classifications in pathology and urology practice (minority opinion). ${ }^{4}$ In particular, they affirmed that:

- The lack of rules for the distinction of G1 from G2 tumours, in addition to the distinction of G2 from G3, in the WHO 1973 bladder cancer classification has resulted in a lumping of cases in the middle. This inevitably leads to a large and heterogeneous group, which is well documented in numerous publications. In spite of its shortcomings, the WHO 1973 bladder cancer classification is probably one of the most extensively used systems, which has given a false impression of validity regarding reproducibility and prognostic relevance. The interobserver reproducibility using the 1973 system is considered to be poor. ${ }^{4}$

- The WHO/ISUP 1998 and the WHO 1999 classifications are congruent and easily "translated" into one another. There are some difficulties in translating the 1973 grades into the new ones.

- Some publications have appeared showing an impact on recurrence rates and rate of progression of the new systems. ${ }^{18-22}$

\section{Recent advances}

Recent reports on the classification and grading schemes of papillary neoplasia are as follows:

- Reproducibility with the WHO/ISUP 1998 and WHO 1999 classifications is worse than with the WHO 1973 scheme. ${ }^{23}$

- The fibroblast growth factor receptor 3 (FGFR3) mutation is among the earliest events in urothelial carcinogenesis- this mutation is already present in a very high proportion of urothelial papillomas. A comparable proportion of mutations is found in papillary urothelial neoplasm of low malignancy potential of the urinary bladder. ${ }^{24}$

- Genetic changes that commonly occur in advanced stage bladder cancer are frequently found in papillary urothelial neoplasm of low malignancy potential of the urinary bladder. ${ }^{25}$

- Samaratunga et al investigated the risk of progression for the WHO/ISUP 1998 and WHO 1973 classifications. ${ }^{26}$ They observed progression in $8 \%$ of patients with PUNLMP and in $11 \%$ of patients with Gl papillary carcinoma (WHO 1973). The same group claim an advantage of the
WHO/ISUP 1998 system over the WHO 1973 system in that the WHO/ISUP 1998 classification recognised a larger proportion of cancers with a poor prognosis. ${ }^{26}$

- Oosterhuis et al found that the prognostic value of the WHO/ISUP 1998 classification is limited, thus questioning the clinical role of this new system in comparison with conventional grading systems. ${ }^{27}$

- The WHO 1973 standard for classification and grading of bladder tumours is a robust, clinically confirmed, widely used, time tested, and reasonably reproducible method for pathological reporting, and is recommended with minor modifications. ${ }^{14}$

\section{INTRAEPITHELIAL FLAT LESIONS}

The most recently revised classification of flat (and endophytic) lesions was published by Lopez-Beltran et al. ${ }^{2}$ This included epithelial abnormalities (reactive urothelial atypia and flat urothelial hyperplasia), presumed preneoplastic lesions and conditions (keratinising squamous and glandular metaplasia, and malignancy associated cellular changes), in addition to preneoplastic (dysplasia) and neoplastic noninvasive (CIS) lesions. Each of these lesions was defined with strict morphological criteria to provide more accurate information to urologists in managing patients. Particular attention was paid to the definition and importance of dysplasia and CIS.

In general, the morphology of dysplasia shows cohesive cells with umbrella cells usually present and characterised by mild nuclear/nucleolar changes that focally include irregular nuclear crowding, and slight hyperchromasia. Anisonucleosis is usually present, and there may be an increased number of cell layers. Nucleoli may be prominent and mitotic figures, when present, are generally basally located. Most cellular abnormalities in dysplasia are restricted to the basal and intermediate layers. Nuclear and architectural features are considered most useful in distinguishing between reactive atypia and dysplasia. Cytokeratin 20 and high molecular weight cytokeratin immunoreactivity might be objective markers of dysplasia in selected cases. ${ }^{28} 29$ To use the term dysplasia without a qualifier is preferable to describe the morphological spectrum of dysplastic lesions of the urothelium.

The morphological diagnosis of CIS requires the presence of severe cytological atypia (nuclear anaplasia); full thickness change is not essential, although it is usually present. Interobserver agreement with CIS is high. The cells of CIS may form a layer that is only one cell thick, of normal thickness (up to seven cells), or the thickness of hyperplasia (greater than seven cells). Prominent disorganisation of cells is characteristic, with loss of polarity and cohesiveness. Superficial (umbrella) cells may be present except in areas of full thickness abnormality. The tumour cells tend to be large and pleomorphic, with moderate to abundant cytoplasm, although they are sometimes small with a high nucleus to cytoplasmic ratio. The chromatin tends to be coarse and clumped. ${ }^{30} 31$ Morphometrically, the cells display increased nuclear area, nuclear perimeter, and maximum nuclear diameter. Nucleoli are usually large and prominent in at least some of the cells, and may be multiple. Mitotic figures are also seen in the uppermost layers of the urothelium, and may be atypical. ${ }^{29} 3233$ Tissue oedema, vascular ectasia, and proliferation of small capillaries are frequent in the lamina propria in cases of CIS.

\section{Controversies}

There are many urothelial "benign" epithelial abnormalities, the identification of which is not always straightforward because of the lack of precise morphological criteria. Some of them represent benign lesions, the morphological identification and interpretation of which do not pose a particular 
problem from the diagnostic point of view. Others are either part of the morphological spectrum of non-papillary neoplasia or reactive lesions, the histological appearance of which cannot be easily distinguished from that of dysplasia. ${ }^{5}$ In such situations morphometrical, immunohistochemical, and molecular techniques should be of great help. For instance, McKenney et al showed that the abnormal expression of cytokeratin 20 (increased), p53 (increased), and CD44 (decreased) in urothelial CIS and the increased expression of CD44 in reactive atypia allows more confident distinction of nonneoplastic urothelial atypia from urothelial CIS. ${ }^{34}$

"There are many urothelial benign epithelial abnormalities, the identification of which is not always straightforward because of the lack of precise morphological criteria"

The use of the term atypia (urothelial atypia) without further specification to encompass reactive atypia is discouraged by Lopez-Beltran et al. ${ }^{2}$ Reactive urothelial atypia is more appropriate. The introduction of the term "atypia of unknown significance $^{\prime \prime 5}$ does not add value in practice. In those rare cases of reactive urothelial atypia in which the pathologist is unsure of whether the changes are reactive or true dysplastic, a conservative approach with repeated cystoscopy and biopsy, after the inflammation has subsided, is indicated.

\section{Recent advances}

The concept of malignancy associated change was introduced by Lopez-Beltran and colleagues ${ }^{2}$ to encompass those epithelial abnormalities that are present in urinary bladders harbouring preneoplastic and neoplastic lesions, and that are not detectable by routine light microscopic examination. ${ }^{35-38} \mathrm{~A}$ few recent studies showed that $50 \%$ of histologically normal urothelium samples adjacent to superficial urothelial carcinoma show genetic anomalies on chromosome 9, similar to the anomalies found in the coexistent carcinoma..$^{35}{ }^{36-41}$ These genetic alterations suggest a neoplastic potential for this flat urothelial lesion, ${ }^{26-38}$ and that additional preneoplastic conditions/lesions not recognisable by light microscopy may be clinically relevant, as shown in the prostate..$^{42}$

Urothelial flat hyperplasia consists of an increase in the number of cell layers, usually 10 or more (but at least more than seven). There are few or no important cytological abnormalities, although slight nuclear enlargement may be focally present. ${ }^{32}{ }^{33}$ Cytologically, this is similar to papillary hyperplasia. Hyperplasia may occasionally be associated with dysplasia or carcinoma in situ in the adjacent mucosa. A recent report found that $71 \%$ of cases of urothelial hyperplasia had the same chromosome 9 deletions seen in coexistent low grade papillary carcinoma. ${ }^{34}$ In contrast, $17 \mathrm{pl} 3$ deletion was found in $8 \%$ of urothelial hyperplasia and low grade carcinoma. ${ }^{37}$ Urothelial hyperplasia has been considered the source of papillary neoplasia. ${ }^{24-46}$

Malignancy associated change and urothelial flat hyperplasia are considered to be the earliest detectable steps in the development of bladder cancer. ${ }^{2}$

\section{MOLECULAR AND GENETIC BASIS OF CLASSIFICATION AND GRADING}

Loss of heterozygosity ( $\mathrm{LOH}$ ) on chromosome 9 is an early event in the generation of superficial bladder cancer, such as G1 and at least part of the spectrum of G2. On the other hand, mutation of p53 represents an early genetic alteration in CIS (and G3). Later on, during progressive growth, p53 also contributes to the carcinogenesis of superficial bladder tumours, whereas LOH on 9p and 9q is involved in the development of CIS to T1-3 tumours. The final progression of superficial carcinomas and CIS seems to require another large

\section{Take home messages}

- Contemporary classification and grading of human papillary urothelial neoplasms remains unsettled. One of the chief motivations for new approaches was to avoid the use of the term "cancer" for neoplasms with a low likelihood of invasion, recurrence, and death

- According to Bostwick and Mikuz, the World Health Organisation (WHO) 1973 classification and grading of bladder tumours is recommended with minor modifications for international use to allow valid comparison of results between different clinical centres. As with all existing classification and grading methods, the WHO classification should be regularly reviewed and updated as appropriate when new and confirmed data emerge

- Molecular pathology could have a role in the further refinements of the classification and grading systems

series of genetic aberrations (fig 2). ${ }^{47}$ The exact sequence of these alterations has not yet been elucidated, even though it is correlated with the morphological classification and grading schemes.

\section{APPENDIX}

The following histological criteria are followed at the Institute of Pathological Anatomy and Histopathology, University of Ancona, to define histologically normal urothelium ${ }^{44}$ :

- Multilayered (for example, five to seven layers) epithelium with two distinctive types of cells: cells of the superficial layer and the underlying intermediate and basal cells.

- The cells comprising all but the superficial layer are small and uniform in size, with well defined borders and amphophylic cytoplasm rich in glycogen. The nuclei are regularly arranged. In particular, they are evenly distributed and polarised towards the surface, and show very finely granular chromatin which accentuates the regularly contoured nuclear borders. Nucleoli are not apparent in tissue fixed in formalin. It is unusual to see mitoses.

- Cells that are much larger than the underlying elements and are often binucleated or multinucleated compose the superficial layer of urothelium. Each cell covers several smaller cells of the immediately underlying layer. The nuclei are large and often show the presence of nucleoli.

\section{ACKNOWLEDGEMENTS}

This research has been partly supported by grants from the University of Ancona and the Italian Ministry of University and Scientific Research.

\section{Authors' affiliations}

R Montironi, R Mazzucchelli, Institute of Pathological Anatomy and Histopathology, Ancona University School of Medicine, I-60020 Torrette, Ancona, Italy

A Lopez-Beltran, Department of Pathology, Reina Sofia University Hospital and Faculty of Medicine, 14071 Cordoba, Spain

D G Bostwick, Bostwick Laboratories, Richmond, and University of Virginia, Charlottesville, VA 23294 Virginia, USA

\section{REFERENCES}

1 Jones PA, Droller M. Pathway of development and progression in bladder cancer: new correlations between clinical observations and molecular mechanisms. Semin Urol 1993;11:177-92.

2 Lopez-Beltran A, Cheng L, Anderson L, et al. Preneoplastic non-papillary lesions and conditions of the urinary bladder: an update based on the Ancona international consultation. Virchows Arch 2002:440:3-11.

3 Bostwick DG, Mikuz G. Urothelial papillary (exophytic) neoplasms. Virchows Arch 2002;441:109-16

4 Busch C, Algaba F. The WHO/ISUP 1998 and WHO 1999 systems for malignancy grading of bladder cancer. Scientific foundation and translation to one another and previous systems. Virchows Arch 2002;441:105-8. 
5 Epstein JI, Amin MB, Reuter V, et al. The bladder consensus conference committee WHO/ISUP consensus classification of urothelial (transitional cell) lesions of the urinary bladder. Am J Surg Pathol 1998;22:1435-48.

6 Mostofi FK, Davis CJ, Sesterhenn IA. Histological typing of urinary bladder fumours. World Health Organisation, international histological classification of tumours, 2nd ed. Berlin: Springer Verlag, 1999.

7 Mostofi FK, Sorbin LH, Torloni H. Histological typing of urinary bladder tumours. International classification of tumours, 19. Geneva: World Health Organisation, 1973

8 Kaubisch S, Lum BL, Reese J, et al. Stage T1 bladder cancer: grade is the primary determinant for risk of muscle invasion. J Urol $1991 ; 146: 28-31$.

9 Takashi M, Sakata T, Murase T, et al. Grade 3 bladder cancer with lamina propria invasion $-\mathrm{pT} 1$ : characteristics of tumor and clinical course. Nagoya J Med Science 1991:53:1-8.

10 Thrasher JB, Frazier HA, Robertson JE, et al. Clinical variables that serve as predictors of cancer-specific survival among patients treated with radical cystectomy for transitional cell carcinoma of the bladder and prostate. Cancer 1994;73:1708-15.

11 Torti FM, Lum BL. Superficial bladder cancer: risk of recurrence and potential role for interferon therapy. Cancer 1987;59:613-20.

12 Malmstrom PU, Busch C, Norlen BJ. Recurrence, progression and survival in bladder cancer. Scand J Urol Nephrol 1987;21:185-95.

13 Montironi R, Mazzucchelli R, Colanzi P, et al. Improving inter-observer agreement and certainty level in diagnosing and grading papillary urothelial neoplasms. Usefulness of a Bayesian belief network. Eur Urol 2002;41:449-57.

14 Bostwick DG. Natural history of early bladder cancer. J Cell Biochem 1999;161(suppl):31-8.

15 Jarkrans T, Vasko J, Bentgsson E, et al. Grading of transitional cell bladder carcinoma by image analysis of histological sections. Anal Cell Pathol 1995;8:135-58

16 Kruger S, Muller H. Correlation of morphometry, nucleolar organizer regions, proliferating cell nuclear antigen and $\mathrm{Ki} 67$ antigen expression with grading and staging in urinary bladder carcinomas. Br J Urol 1995; 75:480-4

17 Montironi R. Ancona international consultation on the diagnosis of non-invasive urothelial neoplasms. Virchows Arch [In press.

18 Alsheikh A, Mohamedali Z, Jones E, et al. Comparison of the WHO-ISUP classification and cytokeratin 20 expression predicting the behavior of low grade papillary urothelial tumors. Mod Pathol 2001; 14:267-72.

19 Cina, S J., Kristen J. Lancaster-Weiss KJ, et al. Correlation of Ki-67 and p53 with the new World Health Organization/International Society of Urological Pathology classification system for urothelial neoplasia. Arch Pathol Lab Med 2001;125:646-51.

20 Cheng L, Newman RM, Bostwick DG. Papillary urothelial neoplasms of low malignant potential. Am Cancer Soc 1999;86:2102-8

21 Desai S, Lim SD, Jimenez RE, et al. Relationship of cytokeratin 20 and CD44 protein expression with WHO/ISUP grade in $\mathrm{pTa}$ and $\mathrm{pT} 1$ papillary urothelial neoplasia. Mod Pathol 2000;13:1315-23.

22 Holmang S, Andius P, Hedelin H, et al. Stage progression in Ta papillary urothelial tumours. Relationship to grade, immunohistochemical expression of tumor markers, mitotic frequency and DNA ploidy. J Urol 2001;165:1124-30.

23 Mikuz G. The reliability and reproducibility of the different classifications of bladder cancer. In: Hauptmann S, Dietel M, Sobrinho-Simões M, eds. Surgical pathology update 2001, 18th European Congress of Pathology. Berlin: ABW Wissenschaftsverlag, 2001:114-5.

24 Van Rhijn BWG, Montironi R, Zwarthoff EC, et al. Frequent FGFR3 mutations in urothelial papilloma. J Pathol 2002;198:245-51

25 Chen L, Zhang S, Ulbright TM, et al. Genetic analysis of papillary urothelial neoplasm of low malignant potential of the urinary bladder using laser-assisted microdissection [abstract]. Mod Pathol 2002;15:158A.
26 Samaratunga H, Makarov DV, Epstein Jl. Comparison of WHO/ISUP and WHO classification of noninvasive papillary urothelial neoplasms for risk of progression. Urology 2002;60:315-19.

27 Oosterhuis JWA, Schapers RFM, Janssen-Heijnen MLG, et al. Histologic grading of papillary urothelial carcinoma of the bladder: prognostic value of the $1998 \mathrm{WHO} / \mathrm{ISUP}$ classification system and comparison with conventional grading system. J Clin Pathol 2002;55:900-5.

28 Harnden P, Eardley I, Joyce AD, et al. Cytokeratin 20 as an objective marker of urothelial dysplasia. Br J Urol 1996:78:870-5.

29 Helpap B, Köllermann J. Assessment of basal cell status and proliferative patterns in flat and papillary urothelial lesions: a contribution of the new WHO classification of urothelial tumors of the urinary bladder. Hum Pathol 2000;31:745-50.

30 McKenney JK, Gomez JA, Desai S, et al. Morphological expressions of urothelial carcinoma in situ. A detailed evaluation of its histologic patterns with emphasis on carcinoma in situ with microinvasion. Am J Surg Pathol 2001;25:356-62

31 Orozco RE, Martin AA, Murphy WM. Carcinoma in situ of the urinary bladder: clues to host involvement in human carcinogenesis. Cancer 1994;76: 115-22.

32 Bostwick DG, Lopez-Beltran A. Bladder biopsy interpretation. New York: United Pathologists' Press, 1999

33 Bostwick DG, Ramnani D, Cheng L. Diagnosis and grading of bladder cancer and associated lesions. Urol Clin North Am 1999;26:493-507.

34 McKenney JK, Desai S, Cohen C, et al. Discriminatory immunohistochemical staining of urothelial carcinoma in situ and non-neoplastic urothelium. An analysis of cytokeratin 20, p53, and CD44 antigens. Am J Surg Pathol 2001;25:1074-8

35 Baithun SI, Naase M, Blanes A, et al. Molecular and kinetic features of transitional cell carcinomas of the bladder: biological and clinical implications. Virchows Arch 2001:438:289-97.

36 Baud E, Catilina P, Boitux JP, et al. Human bladder cancers and normal bladder mucosa present the same hot spot of heterozygous chromosome-9 deletion. Int J Cancer 1998;77:821-4.

37 Hartmann A, Moser K, Kriegmair M, et al. Frequent genetic alterations in simple urothelial hyperplasia of the bladder in patients with papillary urothelial carcinoma. Am J Pathol 1999;154:721-7.

38 Muto S, Horie S, Takahashi S, et al. Genetic and epigenetic alterations in normal epithelium in patients with metachronous bladder cancer. Cancer Res 2000;60:4021-5.

39 Czerniak B, Li L, Chaturvedi V, et al. Genetic modeling of human urinary bladder carcinogenesis. Genes Chromosomes Cancer 2000;27:392-402

40 Hartmann A, Schlake G, Zaak D, et al. Occurrence of chromosome 9 and p53 alteration in multifocal dysplasia and carcinoma in situ of human urinary bladder. Cancer Res 2002;62:809-18.

41 Standler WM, Steinberg G, Yang X, et al. Alterations of the $9 p 21$ and $9 q 33$ chromosomal bands in clinical bladder cancer specimens by fluorescence in situ hybridization. Clin Cancer Res 2001;7:1676-82.

42 Smith G, Elton RA, Beynon LL. Prognostic significance of biopsy results of normal looking mucosa in cases of superficial bladder cancer. Br J Urol 1983;55:665-9

43 Montironi R, Longato Filho A, Santinelli A, et al. Nuclear changes in the normal-looking columnar epithelium adjacent to and distant from prostatic intraepithelial neoplasia and prostate cancer: morphometric analysis in whole-mount sections. Virchows Arch 2000;437:625-34

44 Koss LG. Tumors of the urinary bladder: atlas of tumor pathology, Vol. 11, 2nd series. Washington, DC: AFIP, 1975.

45 Koss LG. Mapping of the urinary bladder: its impact on the concepts of the bladder cancer. Hum Pathol 1979; 10:533-48.

46 Sarma KP. Genesis of papillary tumors: histological and microangiographic study. Br J Urol 1981;55:665-9.

47 Brandau S, Böhle A. Bladder cancer. I. Molecular and genetic basis of carcinogenesis. Eur Urol 2001;39:491-7. 